\title{
Intraosseous transplant of dystrophin expressing chimeric (DEC) cells improves skeletal muscle function in $m d x$ mouse model of Duchenne muscular dystrophy
}

\author{
Mohammad Malik ${ }^{1}$, Maria Siemionow ${ }^{1}$, Joanna Cwykiel ${ }^{1}$, Ahlke Heydemann ${ }^{2}$, Jesus Garcia-Martinez ${ }^{3}$, \\ Krzysztof Siemionow ${ }^{1}$, Erzsebet Szilagyi ${ }^{1}$ \\ ${ }^{1}$ Department of Orthopedics, University of Illinois at Chicago, Chicago, IL, USA \\ 2Department of Physiology and Biophysics, University of Illinois at Chicago, Chicago, IL, USA \\ ${ }^{3}$ Department of Clinical Health Sciences, Saint Louis University, Saint Louis, MO, USA
}

Adv Interv Cardiol 2022; 18, 4 (70): 399-406

DOI: https://doi.org/10.5114/aic.2021.110990

\begin{abstract}
A bstract
Introduction: We previously reported that systemic delivery of dystrophin expressing chimeric (DEC) cells of normal (wt) and dystrophin-deficient ( $m d x)$ myoblast (MB) or mesenchymal stem cell (MSC) origin restored dystrophin expression and improved cardiac function in the $m d x$ mouse model of Duchenne muscular dystrophy (DMD).

Aim: This study evaluated the effect of intraosseous delivery of murine DEC lines of $M B\left(M^{w t} / M^{m d x}\right)$ and $M S C\left(M B^{w t} / M^{m} C^{m d x}\right)$ origin on function of gastrocnemius muscle (GM).

Material and methods: DEC lines created by ex vivo fusion were tested in the mdx mouse model of DMD: Group 1 - vehicle (control), Group 2 - non-fused $0.25 \times 10^{6} \mathrm{MB}^{\text {wt }}$ and $0.25 \times 10^{6} \mathrm{MSC}^{m d x}$ (control), Group 3 - fused $0.5 \times 10^{6} \mathrm{MB}^{\text {wt }} / \mathrm{MB}^{\text {mdx }} \mathrm{DEC}$ and Group 4 - fused $0.5 \times 10^{6} \mathrm{MB}^{w t} / \mathrm{MSC} m d x$ DEC. In situ and in vitro muscle force tests assessed GM function at 90 days post-transplant.

Results: Application of $\mathrm{MB}^{\mathrm{wt}} / \mathrm{MSC}^{m d x}$ and $\mathrm{MB}^{\mathrm{wt}} / \mathrm{MB}^{\operatorname{mdx}} \mathrm{DEC}$ significantly improved the fatigue ratio of GM compared to vehicle-injected controls detected by in vivo muscle force tests $\left(0.567 \pm 0.116, p=0.045\right.$ and $0.489 \pm 0.087, p<0.05$, respectively). MB ${ }^{w t} /$ MSCmdx DEC recipients presented enhanced maximum force at tetanus $(0.145 \pm 0.040 \mathrm{~g} / \mathrm{mg}, p<0.05)$; furthermore, recipients of $\mathrm{MB}^{\mathrm{wt}} / \mathrm{MBmdx} \mathrm{DEC}$ showed a significant increase in the maximum force generation rate compared to vehicle controls $(4.447 \pm 1.090$ $\mathrm{g} / \mathrm{s} / \mathrm{mg}, p<0.05)$. The ex vivo GM force testing in $\mathrm{MB}^{\mathrm{wt}} / \mathrm{MSC} \mathrm{mdx}$ DEC recipients detected increased average GM force compared to vehicle and non-fused controls.

Conclusions: Systemic-intraosseous administration of $\mathrm{MB}^{\mathrm{wt}} / \mathrm{MBm} d x$ and $\mathrm{MB}^{\mathrm{wt}} / \mathrm{MSC} \mathrm{mdx}$ DEC therapy combining the myogenic and immunomodulatory properties of MB and MSC significantly improved skeletal muscle (GM) function of force and resistance to fatigue in an $m d x$ mouse model of DMD.
\end{abstract}

Key words: dystrophin expressing chimeric cells, DEC therapy, Duchenne muscular dystrophy, cell fusion, mesenchymal stem cells, myoblast.

S u m m a ry

We have previously reported that systemic-intraosseous delivery of dystrophin expressing chimeric (DEC) cells of normal $(w t)$ and dystrophin-deficient $(m d x)$ myoblast (MB) or mesenchymal stem cell (MSC) origin restored dystrophin expression and improved cardiac function in the mdx mouse model of Duchenne muscular dystrophy (DMD). Using the same mdx mouse model, this study confirmed that $0.5 \times 10^{6}$ of DEC therapy $\left(\mathrm{MB}^{\text {wt }} / \mathrm{MB}^{m d x}\right.$ and $\left.\mathrm{MB}^{\text {wt }} / \mathrm{MSC}^{m d x}\right)$, which combines the myogenic and/or immunomodulatory properties of MB and MSC, significantly improved the force and resistance to fatigue of skeletal (gastrocnemius) muscles at 90 days after systemic DEC administration as confirmed by standard functional tests.

\section{Introduction}

Duchenne muscular dystrophy (DMD) is an X-linked neuromuscular disorder caused by a mutation in the dys- trophin gene. Dystrophin deficiency results in myofibril damage, inflammation and fibrosis, and manifests as progressive skeletal and cardiac muscle weakness, which

Corresponding author:

Prof. Maria Siemionow, Department of Orthopedics, University of Illinois, United States, phone: +1216212 7012, e-mail: siemiom@uic.edu Received: 12.03.2021, accepted: 28.10.2021. 
subsequently leads to cardiac dysfunction and failure [1-5]. Despite extensive research testing novel cell and gene-based therapies, no cure preventing or treating DMD exists.

We have introduced and tested, in vitro and in vivo, murine and human dystrophin expressing chimeric (DEC) cell lines created by ex vivo cell fusion of normal myoblasts (MB) and normal/dystrophin-deficient MB or mesenchymal stem cells (MSC) for future clinical application to treat DMD [1-3]. Both MB and MSC parent cells used for DEC creation presented desirable characteristics such as regenerative, anti-inflammatory and immunomodulatory potential for development of stem cell-based therapies targeting DMD. We have previously reported that after ex vivo polyethylene glycol (PEG)-mediated cell fusion, the chimeric state of murine and human DEC cells was confirmed by flow cytometry [1-4], confocal microscopy [1, 3, 4], polymerase chain reaction (PCR)-short tandem repeats and polymerase chain reaction-reverse sequence-specific oligonucleotide probe [1, 3, 4]. DEC expressed dystrophin and showed myogenic differentiation [2,3] and decreased immunogenicity $[3,4]$. Moreover, the safety of DEC was confirmed by COMET assay presenting no genotoxicity effect of the fusion procedure on the created DEC line [3, 4].

Our previous study confirmed that intraosseous delivery of murine DEC lines of normal MB (wild type $-w t$ ) fused with dystrophin-deficient $M B\left(M^{m d x}\right)$ or $\left(M^{2} C^{m d x}\right)$ significantly increased dystrophin expression in the cardiac muscle, which correlated with improved cardiac functions of ejection fraction and fractional shortening in $\mathrm{MB}^{w t} / \mathrm{MB}^{m d x}$ and $\mathrm{MB}^{w t} / \mathrm{MSC}^{m d x}$ DEC injected mice [3].

\section{Aim}

This study focused on evaluation of the systemic effect of intraosseous administration of $\mathrm{MB}^{\mathrm{wt}} / \mathrm{MB}^{\mathrm{mdx}}$ and $\mathrm{MB}^{\text {wt }} / \mathrm{MSC}^{\text {mdx }}$ DEC therapies on the function of the skeletal muscle assessed in gastrocnemius muscle (GM) of $m d x$ mice using in situ and ex vivo muscle force testing.

\section{Material and methods}

\section{Experimental animals}

Animal care and experimental protocols were approved by the University of Illinois at Chicago Institutional Animal Care and Use Committee (IACUC), accredited by the American Association for the Accreditation of Laboratory Animal Care (AAALAC).

Six- to eight-week-old male mice - $m d x$ (C57BL/ 10ScSn-Dmdmdx/J, stock number 001801) with the corresponding background snj wild type (wt) mice (C57BL/10ScSnJ, stock number 000476) were purchased from Jackson Laboratories. All animals received humane care with ad libitum access to water and normal chow in compliance with the Principles of Laboratory Animal Care formulated by the National Society for Medical Research and the Guide for the Care and Use of Laboratory Ani- mal Resources published by the US National Institutes of Health.

\section{Ex vivo creation of dystrophin expressing}

chimeric (DEC) cells of myoblast and mesenchymal stem cell origin

The methodology of creating murine $\mathrm{MB}^{\mathrm{wt}} / \mathrm{MB}^{\mathrm{m} d x}$ and $\mathrm{MB}^{w t} / \mathrm{MSC}^{m d x}$ DEC has been reported previously [3, 4]. Briefly, DEC were created via ex vivo polyethylene glycol (PEG)-mediated fusion. The $\mathrm{MB}^{\mathrm{wt}} / \mathrm{MB}^{m d x} \mathrm{DEC}$ line was created from myoblasts isolated from the $m d x$ and snj wild type (wt) mice, whereas the $\mathrm{MB}^{w t} / \mathrm{MSC}^{\mathrm{mdx}} \mathrm{DEC}$ line was created from the wild type $(w t)$ myoblasts and MSC harvested from $m d x$ mice as previously described [3].

\section{Myoblast (MB) isolation}

Minced muscles derived from donor mouse hind limbs were incubated with $1.5 \mathrm{U} / \mathrm{l}$ Collagenase type D (Roche-Thermo Fisher, Waltham, MA, USA) and $2.4 \mathrm{U} / \mathrm{l}$ Dispase II (Sigma, St. Louis, MO, USA) in $2.5 \mathrm{mM} \mathrm{CaCl}_{2}$ solution at $37^{\circ} \mathrm{C}$ for $30 \mathrm{~min}$. Following digestion, muscles were further mechanically dissociated in primary culture medium (F-10+), containing Ham's F-10 medium (Gibco-Thermo Fisher, Waltham, MA, USA), 20\% Fetal Bovine Serum (FBS, Gemini Bio-Products, West Sacrament, CA, USA), $1 \%$ antibiotic-antimycotic solution (Gibco-Thermo Fisher, Waltham, MA, USA) and $2.5 \mathrm{ng} / \mathrm{ml}$ basic fibroblast growth factor (bFGF, PeproTech, Rocky Hill, NJ, USA). Cells filtered through $100 \mu \mathrm{m}$ and $70 \mu \mathrm{m}$ pore size nylon meshes (Thermo Fisher, Waltham, MA, USA) were counted and plated in $75 \mathrm{~cm}^{2}$ collagen-coated tissue flasks (Celltreat Scientific Products, Pepperell, MA, USA). Adherent cells, reaching $60-80 \%$ confluence, were harvested and pre-plated for 15 mina in a collagen-coated flask to eliminate fibroblasts. Non-adherent cells were transferred for further culture in F-10-based Primary Culture Media. After 3-4 additional passages and pre-plating steps, myoblasts (MB) were expanded in F10/DMEM-based Myoblast Growth Medium supplemented with 20\% FBS (Gemini Bio-products, West Sacramento, CA, USA), 1\% antibiotic-antimycotic solution (Gibco-Thermo Fisher, Waltham, MA, USA) and $2.5 \mathrm{ng} / \mathrm{ml}$ bFGF (PeproTech). MB at passages 4-6 were used for the fusion procedure [3].

\section{Mesenchymal stem cell (MSC) isolation}

Bone marrow cells harvested from the femurs and tibias of 6 weeks old $m d x$ mice were washed and cultured in medium containing $40 \%$ alpha Modified Eagle Medium ( $\alpha M E M), 40 \%$ F-12 nutrient mixture (Invitrogen, USA), 10\% FBS (Gemini Bio Products, USA), and 1X antibiotic-antimycotic solution (Invitrogen, USA). After 7-day culturing, the adherent cells were depleted using MACS cell separation columns (Miltenyi Biotec, USA) with antibodies to CD11b and CD45 (eBiosciences, Imc. USA). The CD45-CD11b- subpopulation was cultured in $175 \mathrm{~cm}^{2}$ flasks (Nunc, USA) at 
a density of $1 \times 10^{6}$ cells per flask. MSC at passages $4-6$ were used for the fusion procedure [3].

\section{Ex vivo cell fusion procedure}

The polyethylene glycol (PEG) fusion protocol was performed as previously described [3, 4]. Prior to fusion, parent myoblasts for $\mathrm{MB}^{\mathrm{wt}} / \mathrm{MB}^{\mathrm{mdx}} \mathrm{DEC}$ fusion and myoblasts and MSC for $\mathrm{MB}^{\text {wt }} / \mathrm{MSC}^{\mathrm{mdx}}$ DEC fusion were fluorescently labeled using either PKH26 or PKH67 (Sigma, St. Louis, MO, USA) tracking membrane dyes according to the manufacturer's instructions. Fusion was performed using fusion medium consisting of $3.5 \mathrm{~g}$ of polyethylene glycol (PEG 4000, EMD), $400 \mu \mathrm{l}$ of DMSO (Sigma) and $2 \mathrm{ml}$ of serum-free DMEM medium supplemented with $1 \mathrm{X}$ Antibiotic/Antimycotic solution. Following washing in complete culture medium, the fused cells were transferred to fluorescently activated cell sorting (FACS) buffer containing 5\% HEPES, 1\% EDTA and 5\% FBS. Cells presenting double (PKH26/PKH67) staining were selected by FACS (BD Astrios, BD Biosciences) and transplanted to the recipient $m d x$ mice. The experimental study design is outlined in Figure 1. A total of $n=7$ fusions were performed to create the two murine DEC lines $\mathrm{MB}^{\mathrm{wt}} / \mathrm{MB}^{m d x}$ and $\mathrm{MB}^{\text {wt }} / \mathrm{MSC}^{\mathrm{mdx}}$ and to assess DEC efficacy in vivo after systemic-intraosseous transplant to the $m d x$ mice.

\section{Murine DEC transplantation}

Age-matched 6-8-week-old $m d x$ recipients were randomly assigned to: Group 1 - vehicle injection $(n=6$, $60 \mu \mathrm{PBS}$ ), Group 2 - injection of non-fused $\mathrm{MB}^{\mathrm{wt}}$ and $\operatorname{MSC}^{m d x}\left(n=3,0.5 \times 10^{6}\right.$ in $\left.60 \mu \mathrm{LPBS}\right)$, Group 3 - injection of fused $M^{w t} / M^{w S C}{ }^{m d x}$ DEC $(n=3)$ and Group 4 - injection of $\mathrm{MB}^{w t} / \mathrm{MB}^{m d x} \operatorname{DEC}\left(n=4,0.5 \times 10^{6}\right.$ in $60 \mu \mathrm{l}$ DPBS). Cells were delivered to the right femur of an anesthetized $m d x$ recipient mouse, as previously described [3, 5-7]. Briefly, following skin incision at the mid-femur level, subcutaneous tissue and the overlying muscles were dissected. Next, using a $28 \mathrm{G}$ needle $60 \mu \mathrm{l}$ of bone marrow was aspirated from the recipient's femur medullary cavity and DEC cells or controls suspended in $60 \mu \mathrm{l}$ of sterile saline were administered to the created space using a tuberculin syringe with a $27 \mathrm{G}$ needle (Exelint International, Los Angeles, CA, USA). To prevent cell leakage, the injected bone was sealed with bone wax. The muscle and skin were closed using 4-0 monofilament absorbable suture. Animals recovered in a heated environment
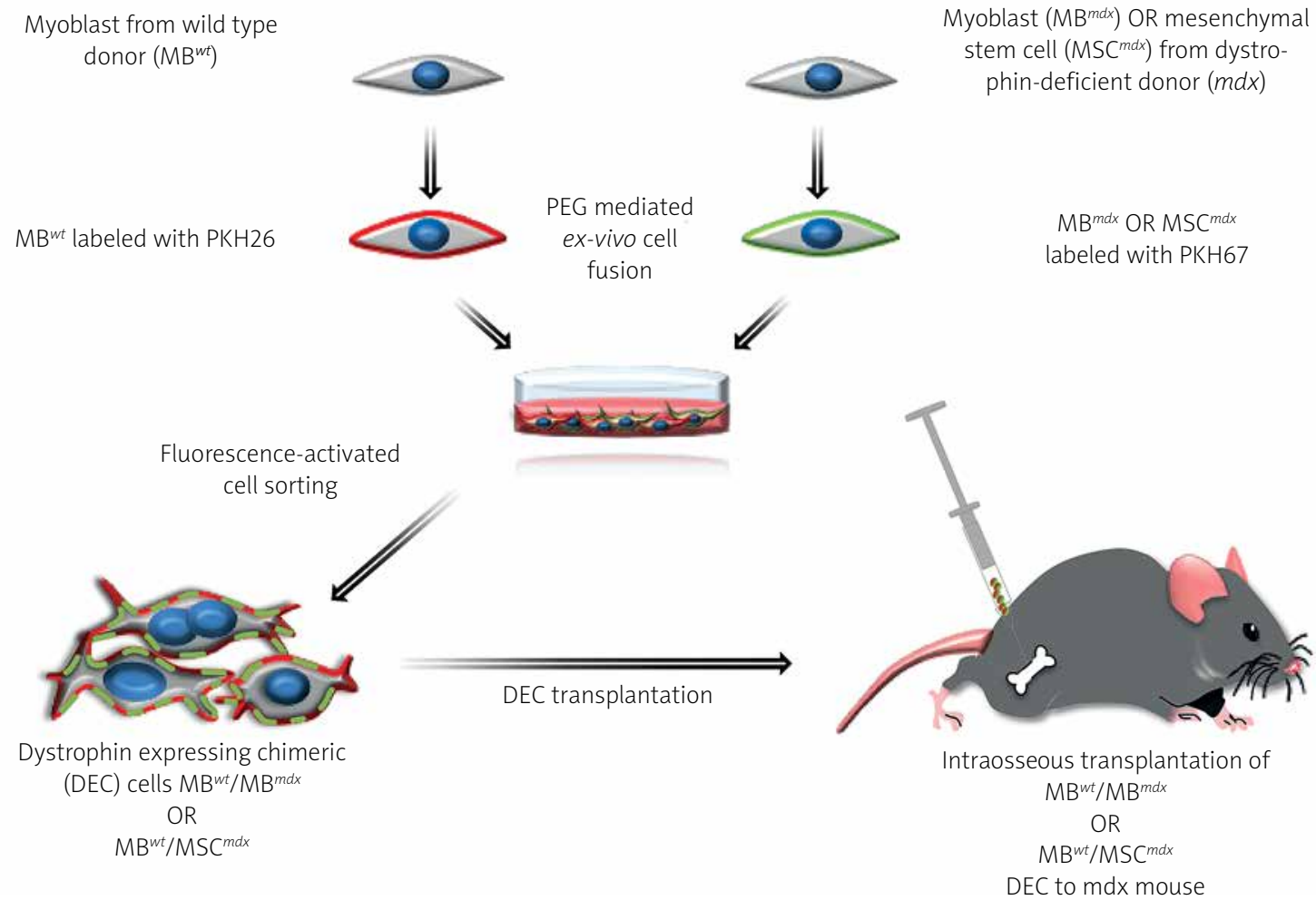

Figure 1. Diagram presenting creation of murine dystrophin expressing chimeric cell (DEC) using polyethylene glycol (PEG) mediated ex-vivo cell fusion procedure and systemic-intraosseous transplantation of murine DEC to the $m d x$ mice. Ex vivo created DEC derived from myoblasts of wild type $\left(\mathrm{MB}^{w t}\right)$ and dystrophin-deficient $m d x$ mice $M^{m} B^{m x}$ origin, or $M^{w t}$ and mesenchymal stem cells of dystrophin-deficient $m d x$ mice $\left(M^{w S C}{ }^{m d x}\right)$. MB ${ }^{w t}$ were labeled with $\mathrm{PKH} 26$ fluorescent dye and $\mathrm{MB}^{m d x}$ or $\mathrm{MSC}^{m d x}$ were labeled with PKH67 fluorescent dye. Following ex-vivo cell fusion DEC cells were sorted based on double positive PKH26/PKH67 labeling and were administered via the systemic-intraosseous route to the femoral bone of an $\mathrm{mdx}$ recipient mouse 
and were returned to the colony. The 90-day follow-up included observation of the DEC injection site, to assess the presence of ecchymosis, inflammation or infection.

\section{Gastrocnemius muscle strength evaluation}

The in vivo and ex vivo gastrocnemius muscle (GM) force measurements were performed at the 90-day endpoint.

\section{In situ force test}

In situ GM force measurements were performed under $1.5 \%$ isoflurane anesthesia. The Achilles tendon was dissected and tied with silk to a force transducer. The sciatic nerve was isolated and stimulated with a bipolar wire electrode. Muscle force was measured after optimal voltage and length were determined. Fatigue was measured after 10 min of submaximal tetanic stimulation as described previously $[1,2,4]$. The GM was kept moist during the whole procedure by continuous drip of Krebs-Henseleit solution (in $\mathrm{mM}$ : $130 \mathrm{NaCl}, 5 \mathrm{KCl}, 1 \mathrm{CaCl}_{2}, 1.1 \mathrm{KH}_{2} \mathrm{PO}_{4}$, $0.85 \mathrm{MgSO}_{4}, 0.6 \mathrm{MgCl}_{2}, 25 \mathrm{HEPES}, 25 \mathrm{NaCO}_{3}, 11$ glucose bubbled with $95 \%$ oxygen and $5 \%$ carbon dioxide). The impact of the drip did not introduce mechanical artifacts. Optimal passive tension was determined by stimulating the sciatic nerve for $6 \mathrm{~s}$. The passive tension was increased every 3 twitches until the maximum force was recorded. Optimal voltage was re-determined after each test. When the optimal voltage changed, the data from the previous test were discarded and the test was repeated. To ensure proper voltage, a set of twitches was elicited beginning at $1.0 \mathrm{~V}$ with a 1-ms pulse every $3 \mathrm{~s}$ with gradual increases in voltage until maximum force was obtained. The voltage used for the experiments was 1.2 times the optimal voltage determined and was usually $2.0 \mathrm{~V}$. After optimal voltage and length were determined, the nerve was stimulated every $3 \mathrm{~s}$ with 1 -ms pulses for 10 repetitions. The amplitude of the twitches and the rates of force generation and relaxation were measured. Twitches were repeated throughout the test to verify that the optimal voltage and passive tension were maintained. A $300-\mathrm{ms}, 50-\mathrm{Hz}$ burst of stimulation was applied to the nerve every $3 \mathrm{~s}$ for 10 min Fatigue is reported as the minimum force, usually at 10 min, as a fraction of the maximum tetanus force. Maximum tetanus typically occurred within the first several seconds of the fatigue experiment, and typically by the second recorded peak. In these measurements, a smaller number means greater fatigue. Potentiation was reported as the maximal force, usually within the first $40 \mathrm{~s}$ of the test, as a percent of the maximum tetanus force $[1,2,4]$.

\section{Ex vivo force test}

The contractile and passive muscle forces of the GM were measured in vitro using the $1200 \mathrm{~A}$ isolated muscle test system (Aurora Scientific, USA). Following $m d x$ recipient euthanasia, the GM dissection including the Achilles tendon from right and left limbs was performed. GMs were placed in $37^{\circ} \mathrm{C}$ Krebs-Henseleit solution in a Radnoti glass chamber tissue bath. Silk ties were applied to attach the Achilles tendon and proximal pole of the muscles to the force transducer. The measurement of GM force was performed after establishing optimal length through a standardized stimuli pattern until reaching maximal wave and maximal strain $[1,2,4]$.

\section{Statistical analysis}

All results are presented as mean \pm SD. Student's $t$-test was used to define statistical significance. $P$-values below 0.05 were considered significant.

\section{Results}

Improvement in gastrocnemius muscle (GM) functional outcomes was confirmed by standard in vivo functional tests assessed at 90 days after systemic-intraosseous DEC transplant to the $m d x$ mice

\section{Muscle force testing}

Muscle force testing showed significant improvement in the fatigue ratios in the $\mathrm{mdx}$ mice injected with $\mathrm{MB}^{\mathrm{wt}} /$ MSC $^{m d x}$ DEC $(0.567 \pm 0.116, p<0.05)$ as well as $\mathrm{MB}^{\text {wt }} /$ $M^{m d x}$ DEC cells $(0.489 \pm 0.087, p=0.015)$ compared to the vehicle controls. In contrast, there was no significant difference between the vehicle controls and $m d x$ mice injected with non-fused cells, which showed a large variability in the response (Figure $2 \mathrm{~A}$ ).

\section{Maximum force at tetanus}

Maximum force at tetanus stimulation, which was standardized for gastrocnemius muscle weights, showed improvement in groups injected with non-fused $M^{\text {wt }}$ and $\mathrm{MSC}^{\mathrm{mdx}}$ cells $(0.113 \pm 0.040 \mathrm{~g} / \mathrm{mg}$ of $\mathrm{GM}$ muscle, $p=0.022)$ and $\mathrm{MB}^{w t} / \mathrm{MSC}^{\mathrm{mdx}}$ cells $(0.145 \pm 0.040 \mathrm{~g} / \mathrm{mg}$ of GM muscle, $p=0.040$ ) compared to the vehicle controls. Although the maximum force was not significantly increased in $m d x$ mice injected with $M^{w t} / M^{m d x}$, there was a trend to an increase as well (Figure 2 B).

\section{Maximum force generation rate and maximum force relaxation}

Maximum force generation rate was significantly increased in $\mathrm{MB}^{w t} / \mathrm{MSC}^{\mathrm{mdx}}$ fused DEC cells compared to vehicle-injected controls $(4.45 \pm 1.1$ vs. $2.7 \pm 0.3, p<$ 0.05 , Figure $3 \mathrm{~A}$ ), and the maximum force relaxation rate showed a significant increase in $\mathrm{MB}^{w t} / \mathrm{MB}^{m d x}$ fused $\mathrm{DEC}$ cells compared to vehicle-injected controls (3.74 \pm 1.95 vs. $1.21 \pm 0.4, p<0.05$, Figure $3 \mathrm{~B}$ ).

\section{Individual twitch amplitudes}

Individual twitch amplitudes did not differ between treatment groups and non-fused and vehicle-injected controls (Figure $3 \mathrm{C}$ ). 
A

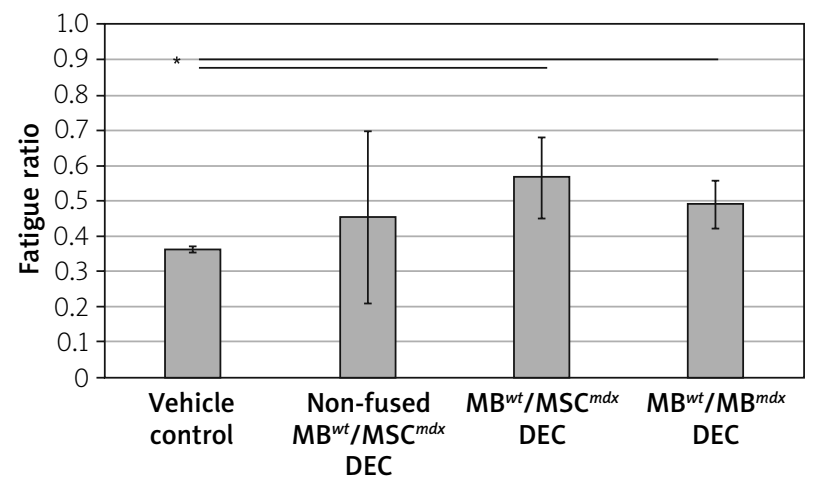

B

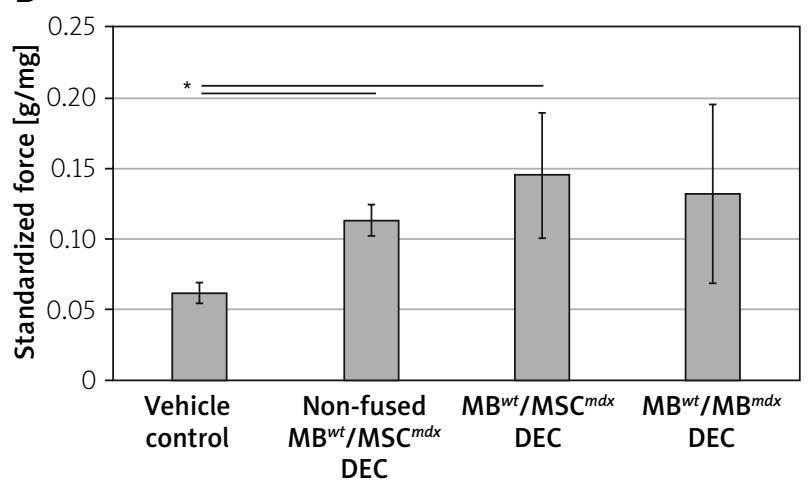

Figure 2. Systemic-intraosseous transplantation of $\mathrm{MB}^{w t} / \mathrm{MB}^{\operatorname{mdx}}$ and $\mathrm{MB}^{\text {wt }} / \mathrm{MSC}^{m d x} \mathrm{DEC}$ lines improved gastrocnemius muscle (GM) fatigue tolerance and force at 90 days after transplant to the $m d x$ mice. In situ GM fatigue tolerance (A) GM force (B). Muscle force was normalized by the isolated GM weight. A smaller number in fatigue tolerance indicates more fatigue. ${ }^{*} P<0.05$

\section{A}

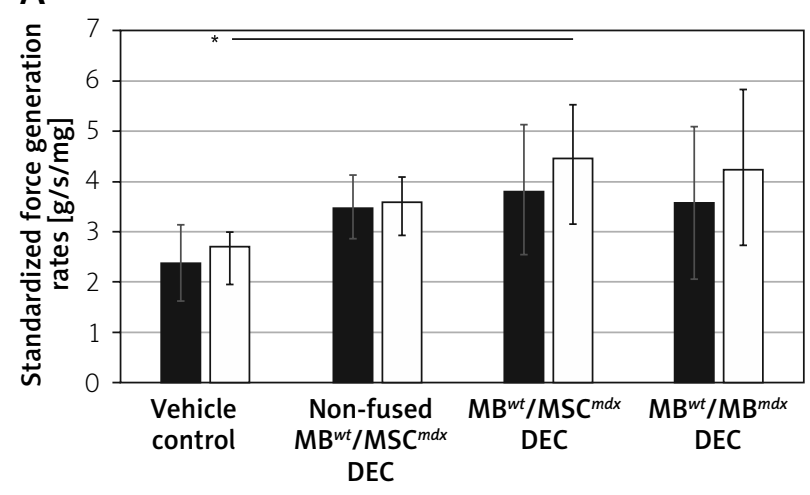

C

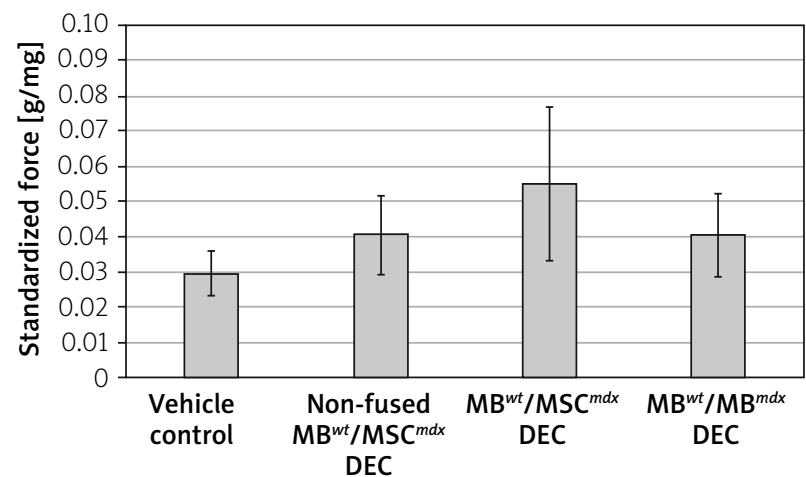

Ex vivo functional outcomes assessed in gastrocnemius muscles (GM) at 90 days after systemic-intraosseous DEC transplant to the $m d x$ mice

The ex vivo GM force testing in $\mathrm{MB}^{\text {wt }} / \mathrm{MSC}^{\text {mdx }} \mathrm{DEC}$ recipients detected an increase in the average GM force compared to the vehicle-injected and non-fused controls ( $32.3 \pm 3.2 \%$ vs. $25 \pm 9.2 \%$, respectively); however, the value was not statistically significant (Figure 4 A). No dif-

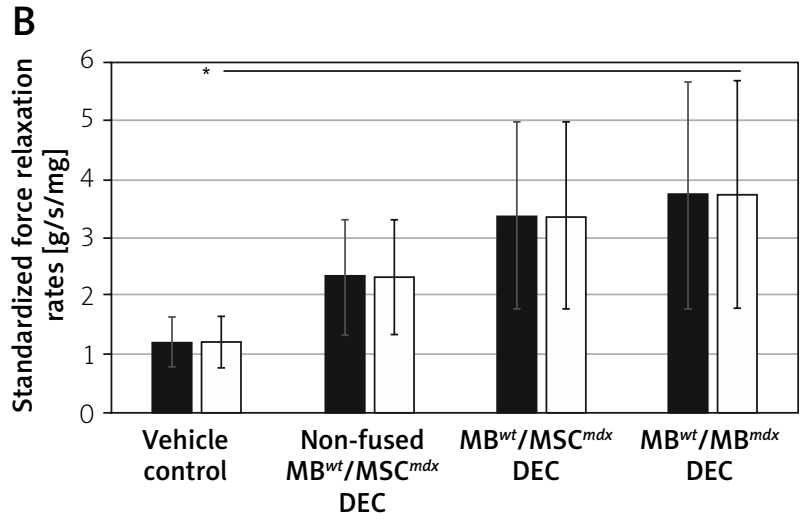

Figure 3. Comparison of in situ tested initial and maximum force generation and relaxation of gastrocnemius muscle (GM) as well as twitch amplitudes at 90 days after $\mathrm{MB}^{w t} / \mathrm{MB}^{m d x}$ and $\mathrm{MB}^{w t} /$ MSC $^{m d x}$ DEC lines after intraosseous administration to $\mathrm{mdx}$ mice. $\mathbf{A}$ - Initial and maximum force generation rates, $\mathbf{B}$ - Initial and maximum force relaxation rates (initial rates of force generation and relaxation are indicated by black bars; maximum rates of force generation and relaxation are indicated by white bars), C - single depolarization twitch amplitudes. Muscle force and twitch amplitudes were normalized by the isolated GM weight. ${ }^{*} P<0.05$

ferences were observed in the fatigue values among the assessed groups (Figures $4 \mathrm{~B}$ and $\mathrm{C}$ ).

\section{Discussion}

Preventing the premature loss of mobility and early mortality of DMD patients through delivery and restoration of muscle dystrophin has been the aim of ongoing development for novel therapies for DMD such as exon skipping, gene editing via viral vectors, and stem cell 
A

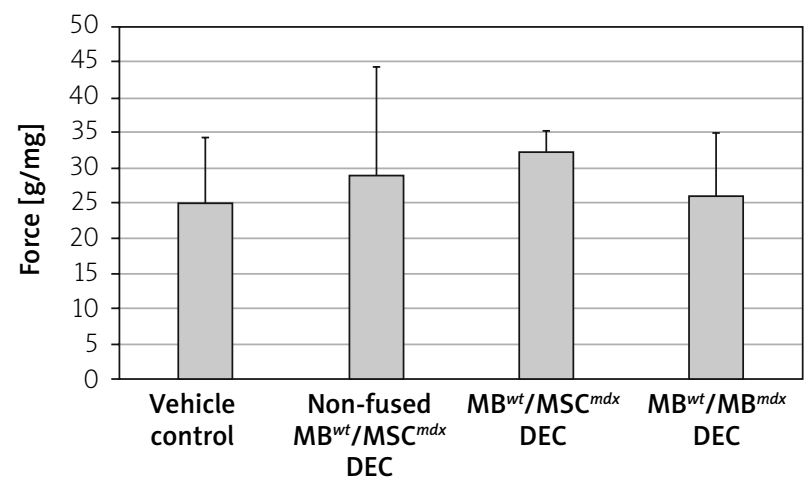

C

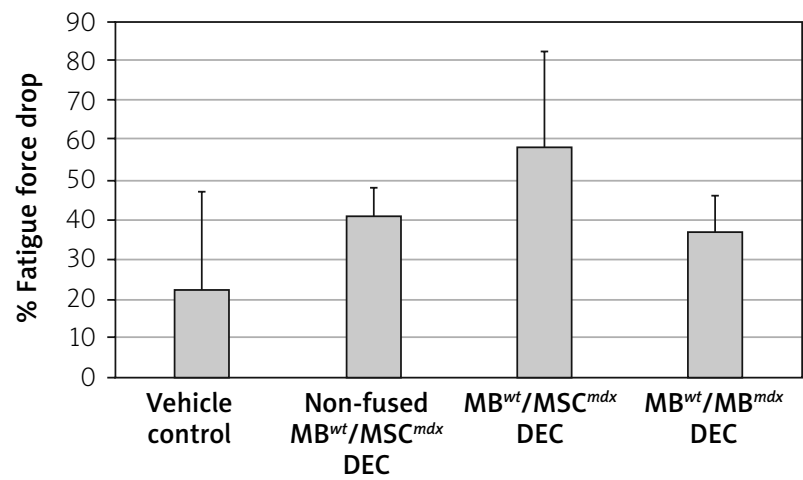

transplants [8-11]. Although MB and bone marrow-based therapies have shown promise, the low engraftment rate caused by immune rejection and the necessity of supportive immunosuppression reduce the efficacy and feasibility of this approach in the clinic [10, 12-14]. However, they fueled the incessant search for new and more effective strategies of stem cell application in treating DMD [15-19]. Previous studies have demonstrated dystrophin expression and restoration of functional dystrophin following transplantation of the autologous cells engineered to express dystrophin via differentiation of pluripotent stem cells to muscle fibers to modify DMD pathology [16].

To address the limitations of current stem cell therapies, we have introduced dystrophin expressing chimeric (DEC) cells as a novel cell-based therapy for DMD. In previous studies, we have confirmed fusion feasibility and performed in vitro phenotype and genotype characterization [1, 2]. In vivo assessment of murine and human DEC confirmed DECs' engraftment, long-term survival and improved skeletal and cardiac muscle function [1-4].

We have also confirmed the therapeutic effect of DEC after intramuscular DEC transplant to the gastrocnemius muscle (GM) of the $m d x$ mice, which revealed over a $37.27 \%$ increase in dystrophin expression, almost two-fold higher than assessed by other laboratories as the level required to obtain functional improvement [2]. These results correlated with trends of improved muscle strength and function assessed by the wire hanging
B

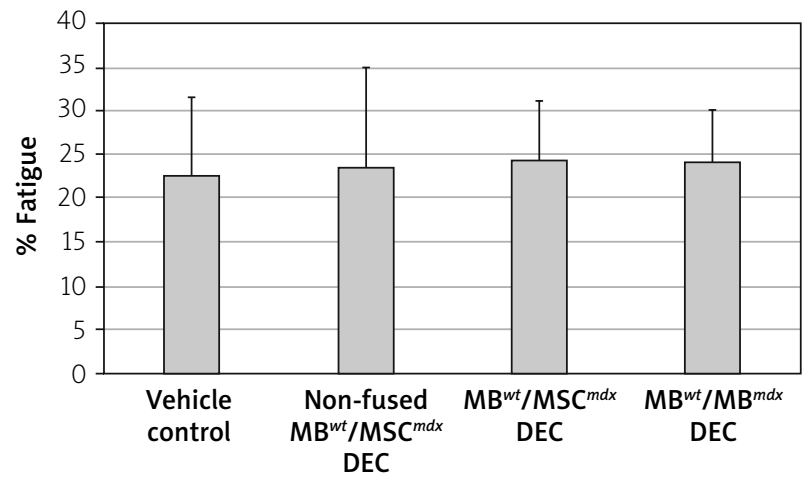

Figure 4. Ex vivo evaluation of (A) muscle force under maximal sine wave stimulation and (B) percentage of fatigue. (C) Fatigue force drop in GM at 90 days after intraosseous administration of $\mathrm{MB}^{\text {wt }} / \mathrm{MB}^{m d x}$ and $\mathrm{MB}^{\text {wt }} / \mathrm{MSC}^{\mathrm{mdx}} \mathrm{DEC}$ lines

test and grip strength measurements up to 30 days after DEC transplant, despite the animal learning and behavioral factors [1, 2]. The current study continues and adds to our previously published data [2, 3] evaluating the effect of murine DEC cell lines of myoblast and MSC origin $\left(\mathrm{MB}^{\text {wt }} / \mathrm{MB}^{m d x}\right.$ and $\mathrm{MB}^{\text {wt }} / \mathrm{MSC}^{\mathrm{mdx}}$, respectively) after systemic-intraosseous transplant to the $m d x$ mice.

Our results suggest that both types of fused DEC lines studied significantly improved the fatigue ratio compared to vehicle-injected controls and non-fused cells, potentially serving as viable novel therapies for improving muscle performance in the DMD pathophysiology cycle. The standardized maximum tetanus force, representing the cumulative performance of all muscle units that comprise the GM during fatigue testing, increased for both treatment groups that received $\mathrm{MSC}^{m d x}$ cells irrespective of fusion status. However, MSC ${ }^{m d x}$ cells would not recover dystrophin production since they are derived from dystrophin-deficient $m d x$ mice. Thus, most likely the MB ${ }^{w t}$ cells were the ones responsible for the increase since they were present in all injections with MSC ${ }^{m d x}$ cells.

Twitch amplitudes for all treatment groups, which represent the muscle's ability to contract in response to a single depolarization event or stimulus, did not differ between study groups. Increases in maximum tetanus force without a corollary increase in twitch amplitude suggest that while muscle units on an individual level may not show clinically impactful improvements in function during a single depolarization, the concerted effort 
between all muscle units with repeat stimulation does demonstrate an amelioration in some of the deleterious effects of dystrophin deficiency. This may be explained by the fact that not all units restored dystrophin to the same extent. To what degree these interactions differ between DEC of myoblast origin $\left(\mathrm{MB}^{\mathrm{wt}} / \mathrm{MB}^{m d x}\right)$ when compared to DEC of MSC origin ( $\mathrm{MB}^{w t} / \mathrm{MSC}^{m d x}$ ) has to be determined and will be a focal point for future studies.

One potential mechanism by which these two DEC lines may differ in their effects on myocytes is via ion channels and ATP pumps in the sarcolemma and sarcoplasm. The speed of membrane depolarization and efficiency of repolarization across the muscle unit is measured as the derivative of the voltage peaks generated by the force transducer, with the upward slopes of peaks representing force generation rates and downward slopes representing force relaxation rates [20]. Mice treated with $\mathrm{MB}^{\mathrm{wt}} / \mathrm{MB}^{\mathrm{mdx}}$ had statistically significantly faster force relaxation rates, suggesting improved efficiency in repolarization compared to other treatment groups. Since $m d x$ myoblasts have already undergone differentiation compared to $m d x$ mesenchymal stem cells, they may have more preexisting cellular infrastructure in their cell membranes such as ion channels, ATP pumps, carrier proteins, etc. Therefore, fusing wild type and $m d x$ myoblasts may result in more ion channels in their resultant shared cell membrane and other proteins involved in relaxation during the excitation-contraction coupling mechanism, leading to a more efficient repolarization response and higher force relaxation rates [20].

Intraosseous injection of both DEC cell lines showed significant improvement in muscle function when compared to vehicle-injected controls and injection of nonfused cells, thus confirming the systemic effect of DEC therapy. These findings are in line with our previous studies confirming better engraftment after intraosseous compared to intravenous transplantation of cell-based therapies [5-7]. Moreover, we reported that the intraosseous delivery route of DEC resulted in protection of cardiac function in the $m d x$ mouse model as confirmed by echocardiography, which revealed the rebound effect on the ejection fraction and fractional shortening observed at 90 days after systemic-intraosseous DEC administration when compared to the vehicle-injected controls [3].

These studies support the intraosseous delivery route as a preferable method for intramuscular delivery of DEC cells, which generate only a local and not a systemic effect due to the limited migration of the locally injected cells. In addition, intraosseous delivery is a short, straightforward procedure which lasts several minutes, whereas intramuscular delivery is a tedious, lengthy procedure under anesthesia, which is less optimal in the clinical scenario when delivered to the pediatric population of DMD patients. Our previous study, evaluating the effect of $\mathrm{MB}^{\mathrm{wt}} / \mathrm{MB}^{m d x}$ and $\mathrm{MB}^{w t} / \mathrm{MSC}^{m d x} \mathrm{DEC}$ therapy on cardiac muscle, confirmed the protective systemic effect of DEC therapy administered via intraosseous injection, and is in line with the results of the current study [4].

It is important to emphasize that functional improvements in gastrocnemius muscle were assessed at 90 days after DEC transplant in the immunocompetent mouse model without immunosuppressive therapy. This further confirms the immunomodulatory effects of DEC cells [4].

In summary, in this study we confirmed the systemic effect of DEC cells of both MB and MSC origin leading to improved function in the skeletal muscle (GM) after intraosseous transplant to the $m d x$ mouse.

\section{Conclusions}

DEC therapy represents a novel concept of ex-vivo fusion of normal and DMD affected myoblasts and MSC to enhance engraftment, elicit immunomodulatory effects, and thus minimize or eliminate the need for immunosuppression. The ultimate goal is to bring DEC therapy closer to clinical application in DMD patients, after verifying the safety and efficacy of DEC treatments with regards to functional outcomes.

\section{Acknowledgments}

This work was supported by the University of Illinois Chancellor's Innovation Fund (CIF) Proof of Concept Award. The authors thank George Rafidi, BS for technical assistance in this study with the in vivo testing.

\section{Conflict of interest}

K.S. is CEO and shareholder of Dystrogen Therapeutics SA; the company holds a license for DEC therapy. M.S. is CMO and shareholder of Dystrogen Therapeutics SA; the company holds a license for DEC therapy. A.H. is the adviser to the Dystrogen Therapeutics. The authors declare a potential conflict of interest. M.S. is the inventor on the patent application filed by the University of Illinois at Chicago. The University of Illinois at Chicago has approved the Nepotism Disclosure and Management Plan for M.S. and K.S. The authors M.M., M.S., J.C., A.H., J.G.M., K.S and E.S. do not have any nonfinancial interest.

\section{References}

1. Siemionow M, Cwykiel J, Heydemann A, et al. Dystrophin expressing chimeric (DEC) human cells provide a potential therapy for Duchenne muscular dystrophy. Stem Cell Rev 2018; 14: 370-84.

2. Siemionow M, Cwykiel J, Heydemann A, et al. Creation of dystrophin expressing chimeric cells of myoblast origin as a novel stem cell based therapy for Duchenne muscular dystrophy. Stem Cell Rev 2018; 14: 189-99.

3. Siemionow $M$, Malik $M$, Langa $P$, et al. Cardiac protection after systemic transplant of dystrophin expressing chimeric (DEC) cells to the mdx mouse model of Duchenne muscular dystrophy. Stem Cell Rev Rep 2019; 15: 827-41. 
4. Siemionow M, Szilagyi E, Cwykiel J, et al., Transplantation of dystrophin expressing chimeric human cells of myoblast/mesenchymal stem cell origin improves function in Duchenne muscular dystrophy model. Stem Cells Dev 2021; 30: 190-202.

5. Siemionow M, Klimczak A, Unal S. Different routes of donor-derived hematopoietic stem cell transplantation for donor-specific chimerism induction across MHC barrier. Transplant Proc 2005; 37: 62-4.

6. Siemionow M, Zielinski M, Ozmen S, et al. Intraosseus transplantation of donor-derived hematopoietic stem and progenitor cells induces donor-specific chimerism and extends composite tissue allograft survival. Transplant Proc 2005; 37: 2303-8.

7. Hivelin M, Klimczak A, Cwykiel J, et al. Immunomodulatory effects of different cellular therapies of bone marrow origin on chimerism induction and maintenance across MHC barriers in a face allotransplantation model. Arch Immunol Ther Exp 2016; 64: 299-310.

8. Kinali M, Arechavala-Gomeza V, Feng L. Local restoration of dystrophin expression with the morpholino oligomer AVI-4658 in Duchenne muscular dystrophy: a single blind, placebo-controlled, dose-escalation, proof-of-concept study. Lancet Neurol 2009; 8: 918-28.

9. Nelson CE, Hakim CH, Ousterout DG. In vivo genome editing improves muscle function in a mouse model of Duchenne muscular dystrophy. Science 2016; 351: 403-7.

10. Skuk D, Tremblay JP. Confirmation of donor-derived dystrophin in a Duchenne muscular dystrophy patient allotransplanted with normal myoblasts. Muscle Nerve 2016; 54: 979-81.

11. Gee P, Xu H, Hotta A. Genome editing, and alternative CRISPR Cas9 technologies for precise gene therapy of duchenne muscular dystrophy. Stem Cells Int 2017; 2017: 8765154.

12. Gussoni E, Bennett RR, Muskiewicz KR, et al. Long-term persistence of donor nuclei in a Duchenne muscular dystrophy patient receiving bone marrow transplantation. J Clin Invest 2002; 110: 807-14.

13. Gussoni E, Blau HM, Kunkel LM. The fate of individual myoblasts after transplantation into muscles of DMD patients. Nat Med 1997; 3: 970-7.

14. Skuk D, Goulet M, Roy B. First test of a "highdensity injection" protocol for myogenic cell transplantation throughout large volumes of muscles in a Duchenne muscular dystrophy patient: eighteen months follow-up. Neuromuscul Disord 2007; 17: 38-46.

15. Sitzia C, Farini A, Jardim L. Adaptive immune response impairs the efficacy of autologous transplantation of engineered stem cells in dystrophic dogs. Mol Ther 2016; 24: 1949-64.

16. Chal J, Oginuma M, Al Tanoury Z. Differentiation of pluripotent stem cells to muscle fiber to model Duchenne muscular dystrophy. Nat Biotechnol 2015; 33: 962-9.

17. Sienkiewicz D, Kulak W, Okurowska-Zawada B, et al., Duchenne muscular dystrophy: current cell therapies. Ther Adv Neurol Disord 2015; 8: 166-77.

18. Skuk D, Tremblay JP. Cell therapy in muscular dystrophies: many promises in mice and dogs, few facts in patients. Expert Opin Biol Ther 2015; 15: 1307-19.

19. Shin JH, Yue Y, Srivastava A, et al. A simplified immune suppression scheme leads to persistent micro-dystrophin expression in Duchenne muscular dystrophy dogs. Hum Gene Ther 2012; 23: 202-9.

20. Tamayo T, Eno E, Madrigal C, et al. Functional in situ assessment of muscle contraction in wild-type and $\mathrm{mdx}$ mice. Muscle Nerve 2016; 53: 260-8. 